\section{Structure of an Acidic Polysaccharide elaborated by Aerobacter aerogenes}

Organisms of the Aerobacter-Klebsiella group have been divided ${ }^{1}$ into many serologically distinct types each of which produces its own type-specific poly. saccharide. Only a few of the structures of these polysaccharides have been elucidated ${ }^{2-4}$ since in general they are of a highly complex nature. Recently we isolated an acidic polysaccharide $(4.45 \mathrm{gm}$.) from the culture medium (38.4 l.) of Aerobacter aerogenes N.C.T.C. 8172 (Klebsiella type 64). The bacteria were grown both in batches and in continuous culture in an apparatus of the type described by Elsworth, Meakin, Pirt and Capells. A peptone-glucose medium was used, the culture was aerated, the $p \mathbf{H ~ 5 . 0}$ and the temperature, $37^{\circ}$. The acidic polysaccharide was freed from neutral polysaccharide by precipitation with 'Cetavlon' 6 and from nucleic acid by the preferen. tial solubility of its cetyl trimethyl ammonium salt in $0.25 M$ sodium chloride. Isolated as its sodium salt, the acidic polysaccharide had C, $45 \cdot 31 ; \mathrm{H}, 6 \cdot 19$; $\mathrm{N}, 0.81 ; \mathrm{P}, 0.11$ per cent and its ultra-violet absorption at $260 \mathrm{~m} \mu$ corresponded to about 1.8 per cent nucleic acid impurity. The polysaccharide contained 28.7 per cent uronic acid (by decarboxylation ${ }^{7}$ ) and the infra-red spectra of the polysaccharide as the free acid and as the sodium salt confirmed the presence of carboxylic acid groups 8 . The polysaccharide showed $[\alpha]_{D}^{17}+29 \cdot 4^{\circ}$ (conc. $0 \cdot 272$ in $M$ sodium chloride).

Acidic hydrolysis of the polysaccharide $(3 \cdot 7 \mathrm{gm}$. with $2 \mathrm{~N}$ sulphurie acid ( 75 c.c.) at $100^{\circ}$ for $3 \mathrm{hr}$. and separation of the neutralized hydrolysate on 'Deacidite $F F^{\prime \prime}$ (carbonate form) gave neutral sugars ( $2 \cdot 3 \mathrm{gm}$.) and acidic sugars ( $1.03 \mathrm{gm}$.$) . Separation of a portion$ $(2.0 \mathrm{gm}$.) of the neutral sugars on a cellulose column irrigated with butanol, ethanol, water, ammonia $(40: 10: 49: 1)$ gave one fraction $(0.295 \mathrm{gm}$.) containing rhamnose and another $(1.5 \mathrm{gm}$.$) containing a$ glucose-mannose mixture. The rhamnose was obtained as crystalline $\alpha$-L-rhamnose, m.p. and mixed m.p. $93-94^{\circ},[\alpha]_{D}^{22}+8 \cdot 2^{\circ}$ equil. (conc. $4 \cdot 13$ in water). The glucose-mannose mixture was separated by treatment ${ }^{9}$ with $p$-nitroaniline in methanol containing hydrochloric acid to give $\mathrm{N}$ - $p$-nitrophenyl- $\beta$-D-glucopyranosylamine dihydrate m.p. and mixed m.p. $184^{\circ}$, $[\alpha]_{D}^{22}-196^{\circ}$ (4 min.) $\rightarrow-203^{\circ}$ (equil.) (conc. 1.02 in dry pyridine), and N-p-nitrophenyl- $\beta$-D-mannopyranosylamine dihydrate m.p. and mixed m.p. $219^{\circ}$, $[\alpha]_{D}^{22}-400^{\circ}\left(4 \mathrm{~min}\right.$.) $\rightarrow-333^{\circ}$ equil. (conc. 0.12 in dry pyridine). Separation of the acidic sugars ( $1 \cdot 0 \mathrm{gm}$.) on a cellulose column irrigated with $n$ butanol-acetic acid-water $(4: 1: 5)$ gave fractions containing hexuronic acid $(0.02 \mathrm{gm}$.$) , aldobiuronic$ acid(s) $(0.41 \mathrm{gm}$.) and mixtures of these $(0.45 \mathrm{gm}$.). The hexuronic acid and its lactone had mobilities on papor chromatograms identical with those of glucuronic acid and glucuronolactone. Reduction of its methyl ester methyl glycoside with lithium aluminium hydride and acidic hydrolysis of the product gave glucose with traces of mannose. This evidence indicated the presence of glucuronic aoid together with a trace of mannuronic acid in the acidic polysaccharide.

Paper chromatographic analysis of an acidic hydrolysate of the aldobiuronic acid fraction showed the presence of unhydrolysed aldobiuronic acid, mannose, glucuronic acid and small amounts of glucose. Similar analysis of a hydrolysate of the reduced aldobiuronic acid fraction showed the presence of approximately equal amounts of glucose and mannose. No rhamnose was detected either in the aldobiuronic acid hydrolysate or that of its reduction product. This evidence indicates that the major component of the aldobiuronic acid fraction was a glucuronosyl-mannose.

The acidio polysaccharide consumed $0.303,0.331$, 0.337 and 0.423 moles of periodate per $162 \mathrm{gm}$. of polysaccharide after oxidation for $48,72,96$ and $110 \mathrm{hr}$. respectively. The corresponding values for formic acid produced were $0.06,0.091,0.1$ and 0.11 . Paper chromatographic analysis of a hydrolysate of the periodate oxidized polysaccharide showed the presence of glucose, mannose and glucuronic acid, but no rhamnose. These results indicate $(a)$ that rhamnose is present either as end groups or linked through the 2-or 4-position, (b) that the maximum number of residues present as end groups or linked $1: 6$ is about 11 per cent, $(c)$ that most of the glueuronic acid residues are not end groups and $(d)$ that large numbers of glucose, mannose and glucuronic acid residues are linked either $1: 3$ or are involved in branching.

One of us (I. R. S.) wishes to thank the British Council for the award of a scholarship under the Colombo Plan.
S. A. Barker
A. B. Foster
I. R. SIDDIQUI
M. STACEY

Chemistry Department,

The University, Edgbaston, Birmingham 15 .

S. J. Pirt

Microbiological Research Establishment, Porton, Wilts. Feb. 6.

1 Edwards. P. R., and Fife, M. A., J. Infect. Diseases, 91, 92 (1952). 2 Heidelberger, M., Goebel, W. F., and Avery, O. T., J. Exp. Med., 42, 727 (1925).

${ }^{3}$ Wilkinson, J. F., Dudman, W. F., and Aspinall, G. O., Biochem. J., 59, 446 (1955)

- Dudman, W. F., and Wilkinson, J. F., Biochem. J., 62, 289 (1956). Elsworth, R., Meakin, L. R. P., Pirt, S. J., and Capell, G. H., J. App. Bact., 19, 264 (1958).

- Jones, A. S., Biochim. Biophys. Acta, 19, 607 (1953).

${ }^{7}$ MeCready, R. M., Swensen, H. A., and Maclay, W. D., Indust. Eng. Chem., 18, 290 (1946).

Barker, S. A., Bourne, E. J., and Whiffen, D. H., Methods of Biochemical Analysis, 3, 213 (1956).

- Weygand, F., Perkow, W., and Kuhner, P., Chem. Ber., 84, 594 (1951)

\section{Colorimetric Detection of Human Caruloplasmin Oxidase Activity after Electrophoresis in Agar Plates or after Immuno-electrophoresis}

THE use of colour reactions combined with electrophoretic analytical methods in agar plates has allowed double (electrophoretic mobility and chemical nature) or triple (electrophoretic mobility, immuno. chemical specificity and chemical nature) characterization of proteins, lipids, glycoproteins and phospholipids in a variety of biological fluids ${ }^{1}$.

Cæruloplasmin, the blue protein of human serum, was isolated in 1948 by Holmberg and Laurell ${ }^{2}$ and characterized as an $\alpha_{2}$-globulin containing copper. They also showed that the substance exhibited oxidase activity with a variety of substrates.

In previous work ${ }^{3}$, we have studied this protein by electrophoretic and immuno-electrophoretic analysis followed by the colorimetric detection in situ of copper-bound cæruloplasmin. In order further to identify cæruloplasmin by another of its inherent 